\title{
Long-term Anabolic-Androgenic Steroid Use Is Associated With Deviant Brain Aging
}

\author{
Astrid Bjørnebekk, Tobias Kaufmann, Lisa E. Hauger, Sandra Klonteig, Ingunn R. Hullstein, and \\ Lars T. Westlye
}

\begin{abstract}
BACKGROUND: High-dose long-term use of anabolic-androgenic steroids (AASs) may cause a range of adverse effects, including brain and cognitive abnormalities. We performed age prediction based on brain scans to test whether prolonged AAS use is associated with accentuated brain aging.

METHODS: T1-weighted magnetic resonance imaging (3D MPRAGE [magnetization-prepared rapid acquisition gradient-echo]) scans were obtained from male weightlifters with a history of prolonged AAS use $(n=130)$ or no AAS use $(n=99)$. We trained machine learning models on combinations of regional brain volumes, cortical thickness, and surface area in an independent training set of 1838 healthy male subjects (18-92 years of age) and predicted brain age for each participant in our study. Including cross-sectional and longitudinal (mean interval = 3.5 years, $n=76$ ) magnetic resonance imaging data, we used linear mixed-effects models to compare the gap between chronological age and predicted brain age (the brain age gap [BAG]) for the two groups and tested for group differences in the rate of change in BAG. We tested for associations between apparent brain aging and AAS use duration, pattern of administration, and dependence.

RESULTS: AAS users had higher BAG compared with weightlifting control subjects, which was associated with dependency and longer history of use. Group differences in BAG could not be explained by other substance use, general cognitive abilities, or depression. While longitudinal analysis revealed no evidence of increased brain aging in the overall AAS group, accelerated brain aging was seen with longer AAS exposure.

CONCLUSIONS: The findings suggest that long-term high-dose AAS use may have adverse effects on brain aging, potentially linked to dependency and exaggerated use of AASs.
\end{abstract}

https://doi.org/10.1016/j.bpsc.2021.01.001

Anabolic-androgenic steroids (AASs) are a family of hormones that comprise testosterone and hundreds of synthetic derivatives of testosterone (1). Administration of supraphysiological doses of AASs in combination with strength training increases lean muscle mass and strength (2). These are desired effects for athletes and bodybuilders, for whom widespread use was seen from the 1950s before it spread to the general population around the 1980s. AAS use has a range of adverse health and social consequences $(3,4)$. Yet, the longterm effects on brain health and cognition are understudied, which is paradoxical given that sex steroids readily pass the blood-brain barrier and affect the central nervous system.

The biological actions of AASs and their metabolites are primarily mediated via the androgen receptors; however, many will also exert physiological effects via estrogen receptor pathways upon aromatization $(5,6)$. Sex steroid receptors are widely expressed in the brain and abundantly expressed in regions such as the brainstem, hypothalamus, amygdala, striatum, hippocampus, and cerebral cortex (7-9). High-dose AAS administration typically involves a complex pattern where testosterone compounds and other AASs are co- administered with doses equivalent to 250 to $5000 \mathrm{mg} / \mathrm{week}$, which is 5 to 100 times greater than the natural male production (10). Administration of supraphysiological doses of AASs suppresses the hypothalamic-pituitary-gonadal axis and reduces the endogenous production of testosterone, luteinizing hormone, and follicle-stimulating hormone. The administration typically continues for several weeks or months, separated by drug-free intervals with the intention to allow the hormonal system to recuperate (11). However, it seems that continuous use persisting for years has become more common (12-16), likely to avoid abstinence symptoms that often occur on cessation $(17,18)$.

While neuroprotective effects of physiological doses of testosterone have been observed $(19,20)$, growing evidence suggests that high-dose long-term AAS use harms the brain. Neurotoxic effects of various sorts of AASs in response to high doses such as those administered by bodybuilders and recreational athletes have been shown (21-26). Moreover, AAS use frequently causes cardiomyopathy $(27,28)$, atherosclerotic disease (27), prolonged hypogonadism (on withdrawal) $(29,30)$, lower low-density lipoprotein cholesterol level (31), and 
impaired insulin sensitivity (32) and occasionally causes toxicity to liver and kidney (33), with potential implications for brain health $(34,35)$.

Emerging evidence from field studies suggests that prolonged high-dose AAS use is associated with aberrant brain aging. For instance, brain imaging has revealed that long-term AAS use is associated with structural, neurochemical (36), and functional brain differences (36-38), including smaller gray matter, cortical, and putamen volume and thinner cerebral cortex (37). In addition, compared with nonusing weightlifters, AAS-exposed weightlifters performed poorer on tests assessing working memory $(12,39,40)$, executive functions $(12,40,41)$, learning and memory $(12,39,41)$, processing speed, and problem solving $(12,40)$. Although such findings are correlational, they have led to the hypothesis that high-dose AAS users are at risk for accelerated brain aging $(42,43)$.

The effects of AAS use show substantial interindividual heterogeneity. Some users exhibit few or no symptoms, while others demonstrate multiple psychological and medical consequences following long-term use $(11,44)$. The range and severity of adverse effects may increase with the burden of use (19) and are particularly pronounced in users fulfilling the criteria for AAS dependence $(1,15,45)$. This includes seemingly more pronounced effects on magnetic resonance imaging (MRI)-based measures of cerebral cortical structure $(37,45)$, self-reported memory problems $(12,41)$, and impaired executive functions $(40)$ and memory functions $(12,39)$ in dependent users. However, group-level differences may disguise substantial individual differences.

Machine learning offers individual predictions based on neuroimaging data (46). For example, training a model to find relationships between brain scans and chronological age allows predicting the age from unseen brain images with high accuracy $(47,48)$. The difference between the predicted age and chronological age, termed the brain age gap (BAG), serves as a surrogate marker of brain health and individual differences in brain maturation and aging $(49,50)$. In adults, an older brain age compared with chronological age has been linked with cognitive impairment (51), cardiovascular risk factors (34), mortality (52), dementia (53), and several other common brain disorders, with regionally differing patterns (54). Conversely, a healthy lifestyle has been associated with a younger-looking brain, with correlations between BAG and level of education and physical activity, as indicated by the number of flights of stairs climbed daily (55). Conversely, drug abuse and addiction have been associated with premature brain aging (56-58) and early onset of agerelated disease (59). While recent studies have documented associations between cumulative exposure to sex hormones and brain age in middle-aged and elderly women (60), the effects of long-term exposure of supraphysiological doses of testosterone and AASs on brain aging have not been studied.

In a sample of 130 AAS users and 99 weightlifting control subjects (WLCs), we used cross-sectional $(n=229)$ and longitudinal $(n=76)$ data to test the hypothesis of higher relative brain age and higher rates of brain aging in AAS users compared with WLCs. We also tested for associations between brain age and AAS use severity, duration, administration (cycling vs. continuous use), and dependence.

\section{METHODS AND MATERIALS}

\section{Participants}

Demographics and clinical characteristics of the sample are summarized in Table 1. The sample is part of a longitudinal study investigating effects of long-term AAS use on brain morphology, cognitive functioning, and emotional processing. Data collection was performed from 2013 to 2015 and from 2017 to 2019 . We recruited male participants engaged in heavy resistance strength training who were either current or previous AAS users reporting at least 1 year of cumulative AAS exposure (summarizing on-cycle periods) or who had never tried AASs or equivalent doping substances. Participants were recruited through websites and forums targeting people partaking in heavy weight training or bodybuilding and through online forums (open and closed) directly addressing AAS use. In addition, posters and flyers were distributed at select gyms in Oslo, Norway. Prior to enrollment, all participants received an information brochure with a complete description of the study. The study was approved by the Regional Committees for Medical and Health Research Ethics-South East Norway, all research was carried out in accordance with the Declaration of Helsinki, and written informed consent was collected from all subjects. The participants were compensated with 1000 Norwegian kroner at time point 1 (TP1) and with 500 Norwegian kroner at time point 2 (TP2).

In total, 139 AAS users and 109 WLCs underwent brain MRI. Of these, 19 participants were excluded. Among AAS users, 2 participants did not fulfill the inclusion criteria of at least 1 year cumulative exposure, 1 participant was excluded owing to a previous head injury that had caused coma, 1 participant was excluded owing to poor scan quality, 2 participants were excluded owing to IQs $<80$, and 2 participants were excluded owing to missing background information. Among WLCs, 1 participant was excluded owing to epilepsy, 2 participants did not match the AAS group on strength training regimens, and 3 participants were excluded owing to missing background information. Furthermore, 3 WLCs were excluded owing to clinically significant abnormalities based on a neuroradiological examination. In addition, 1 73-year-old AAS user and 175 year-old WLC were excluded owing to their substantially higher age than the rest of the sample, which may influence the brain age models and findings. Therefore, our final sample comprised 130 AAS users and 99 WLCs. Among those, 36 AAS users and 40 WLCs were scanned at TP2, on average 3.5 years after TP1.

\section{Image Acquisition}

MRI was performed using a 3T Siemens Skyra scanner (MAGNETOM Skyra; Siemens, Erlangen, Germany) equipped with a 20-channel head coil. Anatomical 3D T1-weighted MPRAGE (magnetization-prepared rapid acquisition gradientecho) sequences with the following parameters were used for volumetry and cortical surface analyses: repetition time $=$ $2300 \mathrm{~ms}$, echo time $=2.98 \mathrm{~ms}$, inversion time $=850 \mathrm{~ms}$, flip angle $=81^{\circ}$, bandwidth $=240 \mathrm{~Hz} /$ pixel, field of view $=256 \mathrm{~mm}$, voxel size $=1.0 \times 1.0 \times 1.0 \mathrm{~mm}, 176$ sagittal slices, and acquisition time $=9: 50$ minutes. Scan quality was inspected at the scan session, and scans were rerun in case of movement. 
Table 1. Demographics, Sports Information, Substance Use, and Use of Psychopharmaca

\begin{tabular}{|c|c|c|c|c|c|c|c|c|}
\hline \multirow[b]{2}{*}{ Sample Characteristics } & \multicolumn{2}{|c|}{ WLCs $(n=99)$} & \multicolumn{2}{|c|}{$\begin{array}{l}\text { AAS Users } \\
(n=130)\end{array}$} & \multirow[b]{2}{*}{$t$} & \multicolumn{2}{|c|}{$\begin{array}{l}\text { 95\% Confidence } \\
\text { Interval }\end{array}$} & \multirow[b]{2}{*}{$p$} \\
\hline & Mean & SD & Mean & SD & & LL & UL & \\
\hline \multicolumn{9}{|l|}{ Demographics } \\
\hline Age, years & 35.0 & 8.8 & 36.2 & 9.4 & -0.97 & -3.6 & 1.2 & .332 \\
\hline Education, years $^{a}$ & 16.3 & 2.5 & 14.4 & 2.7 & 5.51 & 1.3 & 2.7 & $<.001$ \\
\hline $\mathbb{I Q}^{b}$ & 115.4 & 9.2 & 106.0 & 10.9 & 7.06 & 6.9 & 12.3 & $<.001$ \\
\hline Alcohol, units/week ${ }^{c}$ & 3.5 & 4.7 & 2.6 & 3.3 & 1.45 & -0.3 & 2.1 & .148 \\
\hline Height, cm & 180.3 & 12.3 & 181.4 & 6.8 & -0.88 & -3.7 & 1.4 & .378 \\
\hline Weight, kg & 90.6 & 11.7 & 97.7 & 15.2 & -3.85 & -10.7 & -3.5 & $<.001$ \\
\hline Body mass index & 28.7 & 11.9 & 29.7 & 4.2 & -0.88 & -3.2 & 1.2 & .379 \\
\hline Strength training, $\mathrm{min} / \mathrm{week}^{d}$ & 399.0 & 223.2 & 360.9 & 226.7 & 1.22 & -23.4 & 99.6 & .223 \\
\hline Endurance training, $\mathrm{min} / \mathrm{week}^{d}$ & 98.0 & 129.2 & 122.9 & 183.3 & -1.15 & -67.4 & 17.6 & .25 \\
\hline \multirow[t]{2}{*}{ Bench maximum, $\mathrm{kg}^{\mathrm{e}}$} & 138.3 & 30.7 & 171.9 & 33.6 & -7.70 & -42.3 & -25.0 & $<.001$ \\
\hline & \multicolumn{2}{|c|}{$\%$} & \multicolumn{2}{|c|}{$\%$} & $\chi^{2}$ & & & $p$ \\
\hline \multicolumn{9}{|l|}{ Training Classification $^{f}$} \\
\hline Bodybuilding/fitness & \multicolumn{2}{|c|}{18.9} & \multicolumn{2}{|c|}{44.8} & 15.8 & & & $<.001$ \\
\hline Weightlifting & \multicolumn{2}{|c|}{26.3} & \multicolumn{2}{|c|}{19.8} & 1.3 & & & .363 \\
\hline Combat sports & \multicolumn{2}{|c|}{20.2} & \multicolumn{2}{|c|}{23.1} & 0.7 & & & .414 \\
\hline Recreational exercise & \multicolumn{2}{|c|}{30.5} & \multicolumn{2}{|c|}{32.8} & 0.1 & & & .729 \\
\hline Psychopharmaca $^{g}$ & \multicolumn{2}{|c|}{7.2} & \multicolumn{2}{|c|}{35.0} & 23.6 & & & $<.001$ \\
\hline Smoker $^{g}$ & \multicolumn{2}{|c|}{1.0} & \multicolumn{2}{|c|}{13.6} & 11.3 & & & .001 \\
\hline
\end{tabular}

AAS, anabolic-androgenic steroid; LL, lower limit; UL, upper limit; WLC, weightlifting control subject.

${ }^{a-f}$ Data availability for the different measures varied to some degree. Mean values are based on the number of nonexposed/AAS-exposed participants: $98 / 124^{a}, 99 / 128^{b}, 89 / 91^{c},>95 / 114^{d}, 96 / 119^{e}, 98 / 118^{f}$.

${ }^{g}$ Fisher's exact test was applied because of too few responses for a category.

\section{MRI Processing and Brain Age Estimation}

All datasets were processed using FreeSurfer version 5.3 (https://surfer.nmr.mgh.harvard.edu) (61), and segmentations and reconstructions were visually inspected and edited if needed.

A machine learning model was trained to predict brain age based on volume, area, and thickness data following a recent implementation (54). The training set for brain age estimation included MRI scans from 1838 healthy male subjects from different cohorts (mean age $=46 \pm 20$ years, age range $=$ 18-92 years) obtained from several publicly available datasets and processed in the same pipeline. The age distributions for the training set and our cohort are shown in Figure 1A, and information about included datasets is shown in Table S1. The MRI features were derived from the Human Connectome Project cortical parcellation (62), comprising 180 regions of interest per hemisphere for thickness, area, and volume. In addition, we used subcortical and cerebellar volumes from FreeSurfer. The full set comprised 1118 features in total. We used the extreme gradient boosting package xgboost in $\mathrm{R}$ to train machine learning models for brain age estimation. In line with recent work, the learning rate was preset to eta $=0.01$ and the optimal number of rounds (nrounds) was determined in a nested cross-validation loop (54).

For all participants, brain age and BAG were estimated using features from either the whole brain or subregions $(54,63)$, including occipital, frontal, temporal, parietal, cingulate, insular, and cerebellar/subcortical features, based on the lobe parcellation labels from FreeSurfer (61). We corrected for a well-known bias in age prediction (64) using a procedure described in (65). Briefly, the association between BAG and age was estimated using linear models including relevant covariates, and the resulting parameter estimate reflecting the linear association between BAG and chronological age was used to adjust the individual brain age estimates prior to recalculation of BAG.

\section{Interviews and Screening Instruments}

Demographics and clinical data were assessed using a selfreport questionnaire and a semistructured interview. Current and previous non-AAS substance use were assessed with the Alcohol Use Disorders Identification Test (66), the Drug Use Disorders Identification Test (67), and the drug and alcohol dependence scales from the Millon Clinical Multiaxial Inventory-III, where a composite score of substance use was computed from the mean scores of these $z$-transformed subtests. The depression scale from the Millon Clinical Multiaxial Inventory-III was used to covary for depressive symptoms. Total lifetime AAS dose ingested was calculated as the lifetime average weekly dose reported and lifetime weeks of AAS exposure, in line with previous studies $(1,68,69)$. IQ was assessed using the Wechsler Abbreviated Scale of Intelligence (70).

\section{Doping Analysis}

Urine samples were collected and analyzed for AASs and some antiestrogens using gas and liquid chromatography coupled to mass spectrometry at the World Anti-Doping 


\section{A Training set}

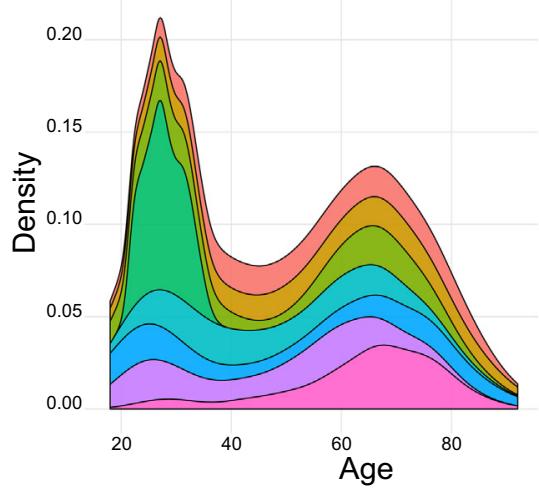

$\square$ CAMCAN $\square$ ds000222 $\square$ IX $\square$ DLBS



Test set

B

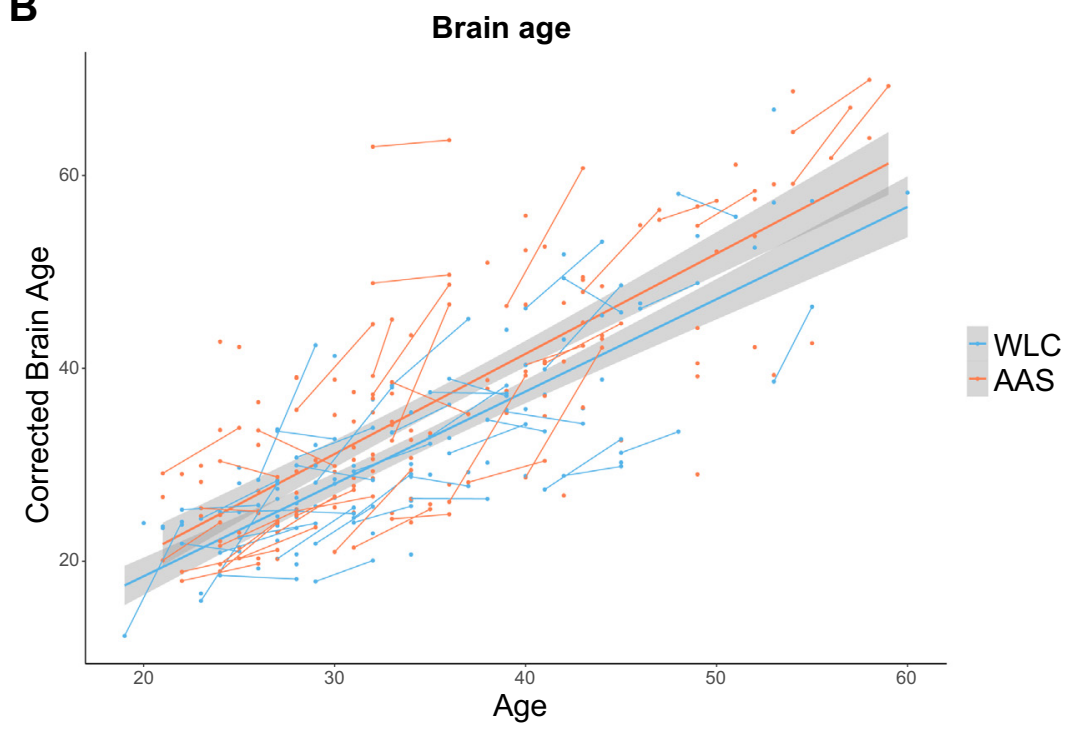

Figure 1. Age distribution and predicted brain age as a function of age. (A) Age distributions for the training set and our cohort. (B) Predicted global brain age corrected for age as a function of chronological age. The fit lines represent the best linear fit within each group, and the points connected by lines represent individual change in brain age gap between the two magnetic resonance imaging scans for each individual. AAS, anabolic-androgenic steroid users; WLC, weight-lifting control subjects.
Agency accredited Norwegian Doping Laboratory at Oslo University Hospital (71). The criteria used to determine AAS use were 1) urine samples positive for AAS compounds and 2) a testosterone/epitestosterone (T/E) ratio $>15$ equivalent to previous work $(37,71)$. Other compounds, including stimulants and remaining antiestrogens, were analyzed with liquid chromatography and mass spectrometry.

\section{Statistical Analysis}

Group differences in demographic data were evaluated with two-tailed independent-samples $t$ tests and $\chi^{2}$ and Fisher's exact tests for categorical data. To assess group differences in global and regional BAG, linear mixed-effects (LME) models were tested using the Ime function in the $R$ (72) package Ime4 (73). In fitting the model, we entered TP and age as fixed effects. Participant ID was entered as a random effect (intercept). Visual inspection of residual plots did not reveal obvious deviations from homoscedasticity or normality.
The significance threshold was set at $p<.05$, corrected for multiple comparisons using false discovery rate (FDR) adjustment (74).

Sensitivity Analyses. Similar LME models including a group by time interaction were run to test for differences in the rate of change between AAS users and WLCs. In addition, to test for confounding effects of cognitive ability, depression, and non-AAS substance use, the main analyses were rerun including IQ, depressive scores, and a composite score of non-AAS substance use as additional covariates. Because we were primarily interested in long-term exposure, and because stricter inclusion criteria have previously been applied $(29,32)$, we reran the main analyses after including only AAS users with more than 2 years of AAS use.

Next, similar LME analyses were conducted to test for differences between subgroups of AAS users: 1) use category: WLCs and AAS users fulfilling the criteria for AAS dependence 
and nondependent users; 2) use pattern: WLCs and AAS users practicing a continuous way of administrating AASs versus users administrating AASs in cycles; 3) use state: WLCs and current and previous AAS users; and 4) use length: AAS users with $<10$ years of exposure and users with $\geq 10$ years' history of AAS exposure.

Lastly, because only $\sim 50 \%$ of the sample took part at TP2, we conducted linear models controlling for age to examine whether BAG at baseline was associated with study dropout.

\section{RESULTS}

\section{Demographics and User Characteristics}

Table 1 summarizes key clinical and demographic characteristics. Years of education and IQ were higher among WLCs, and AAS users were heavier and stronger than WLCs. The use of prescribed psychotropic medication was significantly higher among AAS users, where antidepressants and anxiolytics were the typical preparations prescribed (not shown). The majority of users $(65 \%)$ and nonusers (93\%) reported no previous or current use of prescribed psychotropic medication.

\section{Characteristics of AAS Use}

The average duration of AAS use at baseline was 10.6 years $(\mathrm{SD}=7.7)$, and the mean age of onset was 22 years $(\mathrm{SD}=6.6$, range $=12-52$ ). Mean weekly AAS dose was $1023 \mathrm{mg}(\mathrm{SD}=$ 656 , range $=100-3750$ ), and mean calculated lifetime dose was $444 \mathrm{~g}(\mathrm{SD}=452$, range $=20-2016)$. Continuous AAS administration was reported by 43 users (33.1\%), and $78(60.0 \%)$ reported a cycling pattern, rotating between periods on and off AASs. The remaining 9 users $(6.9 \%)$ were on testosterone replacement therapy, had missing details regarding administration pattern, or were difficult to classify. In addition, 77 AAS users (59.2\%) fulfilled the criteria for AAS dependence and 87 $(67 \%)$ had used AASs within the past 6 months and were defined as current users. Current users had a longer history of AASs and a higher age of onset compared with past AAS users. No differences in extent of use measures were seen between cyclical and continuous users, whereas dependent users had used longer, debuted earlier, and used higher AAS doses compared with nondependent users (Table S2).

None of the 99 WLCs tested positive for AASs or had a T/E ratio above threshold, whereas tests indicative of AAS use were seen in $78.2 \%(n=68)$ of current users and in $7.5 \%(n=3)$ of previous users. The positive tests among previous users could be compatible with previous use, stated by the participants, and one test with elevated T/E ratio was consistent with reported medical use of testosterone replacement therapy. The mean $\mathrm{T} / \mathrm{E}$ ratios for the groups were $1.4(\mathrm{SD}=1.6$, range $=$ $0.1-10.0)$ for WLCs $(n=99), 44.8(\mathrm{SD}=50.6$, range $=$ $0.1-226.0)$ for current users $(n=82)$, and 2.8 (SD = 7.9, range $=0.0-50.4)$ for previous users $(n=39)$, where previous users and WLCs were significantly different from current users $\left(t_{220}=-7.2, p<.001\right)$. The frequencies of the specific AASs found in the urine samples are summarized in Figure $S 1$ along with a summary of the most popular compounds based on self-reports.

\section{Brain Age Prediction}

A 10-fold cross-validation on age prediction in the training set confirmed high accuracy of the model, with correlations between chronological age and predicted age ranging from

Table 2. Main Model

\begin{tabular}{|c|c|c|c|c|c|c|c|c|}
\hline & Full Brain & Frontal & Temporal & Insula & Cingulate & Parietal & Occipital & Subcortical \\
\hline \multicolumn{9}{|l|}{ Group AAS } \\
\hline Fixed-effects estimate $(\beta)$ & $3.288^{a}$ & $3.743^{b}$ & $2.573^{c}$ & $2.483^{c}$ & $2.613^{C}$ & $2.033^{C}$ & $2.885^{c}$ & 1.906 \\
\hline SE & 0.918 & 1.177 & 1.046 & 0.979 & 1.185 & 1.025 & 1.154 & 1.064 \\
\hline$t$ statistic & 3.580 & 3.180 & 2.460 & 2.535 & 2.205 & 1.984 & 2.499 & 1.790 \\
\hline FDR-corrected $p$ & $<.001$ & .008 & .024 & .024 & .037 & .055 & .024 & .075 \\
\hline \multicolumn{9}{|l|}{ Time } \\
\hline Fixed-effects estimate $(\beta)$ & 0.260 & 0.221 & -0.846 & -0.879 & -0.436 & 0.008 & 0.874 & 0.796 \\
\hline SE & 0.513 & 0.720 & 0.563 & 0.552 & 0.685 & 0.554 & 0.701 & 0.522 \\
\hline$t$ statistic & 0.507 & 0.307 & -1.504 & -1.592 & -0.636 & 0.015 & 1.246 & 1.527 \\
\hline FDR-corrected $p$ & .817 & .867 & .36 & .36 & .817 & .988 & .432 & .36 \\
\hline \multicolumn{9}{|l|}{ Age } \\
\hline Fixed-effects estimate $(\beta)$ & -0.021 & -0.007 & -0.033 & -0.020 & 0.007 & -0.015 & -0.025 & -0.056 \\
\hline SE & 0.050 & 0.064 & 0.056 & 0.053 & 0.064 & 0.055 & 0.062 & 0.057 \\
\hline$t$ statistic & -0.415 & -0.105 & -0.590 & -0.385 & 0.113 & -0.280 & -0.394 & -0.975 \\
\hline FDR-corrected $p$ & .916 & .916 & .916 & .916 & .916 & .916 & .916 & .916 \\
\hline Observations & 305 & 305 & 305 & 305 & 305 & 305 & 305 & 305 \\
\hline Log Likelihood & -986.417 & $-1,070.714$ & $-1,022.649$ & $-1,007.022$ & $-1,067.389$ & $-1,016.988$ & $-1,064.043$ & $-1,019.256$ \\
\hline Akaike Information Criterion & $1,984.834$ & $2,153.427$ & $2,057.298$ & $2,026.044$ & $2,146.777$ & $2,045.976$ & $2,140.085$ & $2,050.512$ \\
\hline Bayesian Information Criterion & $2,007.155$ & $2,175.749$ & $2,079.620$ & $2,048.366$ & $2,169.099$ & $2,068.298$ & $2,162.407$ & $2,072.834$ \\
\hline
\end{tabular}

The upper section shows linear mixed-effects model results for estimates of brain age gaps for full brain and subregions. Group levels: weightlifting control subjects (reference, $n=139)$ and AAS users $(n=166)$.

AAS, anabolic-androgenic steroids; FDR, false discovery rate.

${ }^{a} p$ (uncorrected) $<.001$.

${ }^{b} p$ (uncorrected) $<.01$.

${ }^{c} p$ (uncorrected) $<.05$. 
$r=.93$ (mean absolute error $=5.76$, root mean squared error $=$ 7.57) for the global model to $r=.76$ (mean absolute error $=$ 10.05 , root mean squared error $=12.94$ ) for the model based on occipital features (Table S3). Figure 1 shows predicted age plotted as a function of chronological age for the test set of AAS users and WLCs, and Table S3 summarizes the prediction accuracies.

\section{Associations Between AAS Use and BAG}

Table 2 summarizes the results from the LME models. Significant main effects for group were found for the global BAG $\left(\beta_{305}=3.29, t=3.58, p_{\mathrm{FDR}}<.001\right)$ and for the frontal, temporal, insular, cingulate, and occipital BAGs. An examination of the fixed-effects estimates showed higher BAG in AAS users compared with WLCs in all regions. There were no significant main effects of time or age.

When including an interaction term between group (and subgroups of AASs) and time, few significant main effects were found (Tables S4-S8). One global BAG model survived FDR correction and revealed a significant group by time interaction, indicating accelerated aging in users with $\geq 10$ years of use compared with WLCs $\left(\beta_{305}=3.68, t=3.06, p_{\mathrm{FDR}}=.024\right)$ (not displayed in Table S8). The longitudinal findings are depicted in Figure 2 for the global BAG.

\section{Sensitivity Analyses}

Sensitivity analyses revealed that the main effect of group remained significant for the global BAG when IQ, non-AAS substance use, and depression were included as fixed effects in the model (Table 3). Frontal and subcortical BAG differences were found at an FDR-corrected threshold of $p<.05$, whereas group differences for the temporal, insula, cingulate, and occipital models were no longer significant when adding covariates. Furthermore, the sensitivity analyzes omitting AAS users with $<2$ years of AAS use revealed significant main effects of group, with higher BAG for AAS users in all but the subcortical models (Table S9).

Sensitivity analyses with WLCs and subgroups of AAS users revealed significant main effects of use category with higher BAG in dependent AAS users compared with WLCs for all regions, whereas nondependent AAS users showed no significant differences from WLCs (Table S10). Significant main effects were also found for use pattern with higher BAG in full brain and some regional models for cyclic and continuous AAS administration compared with WLCs (Table S11). In addition, significant main effects of use state were seen, where current AAS users had significantly higher BAG in most regions compared with WLCs (Table S12). Previous users ( $>6$ months since last use) were not significantly different from WLCs, although differences were seen at an uncorrected significance level for some models including the full-brain measure $\left(\beta_{302}=2.55, t=2.24, p=\right.$ .03). Lastly, splitting the groups into shorter history $(<10$ years) versus longer history ( $\geq 10$ years) of AAS use revealed significant main effects of use length and higher BAG compared with WLCs for the full-brain model and some
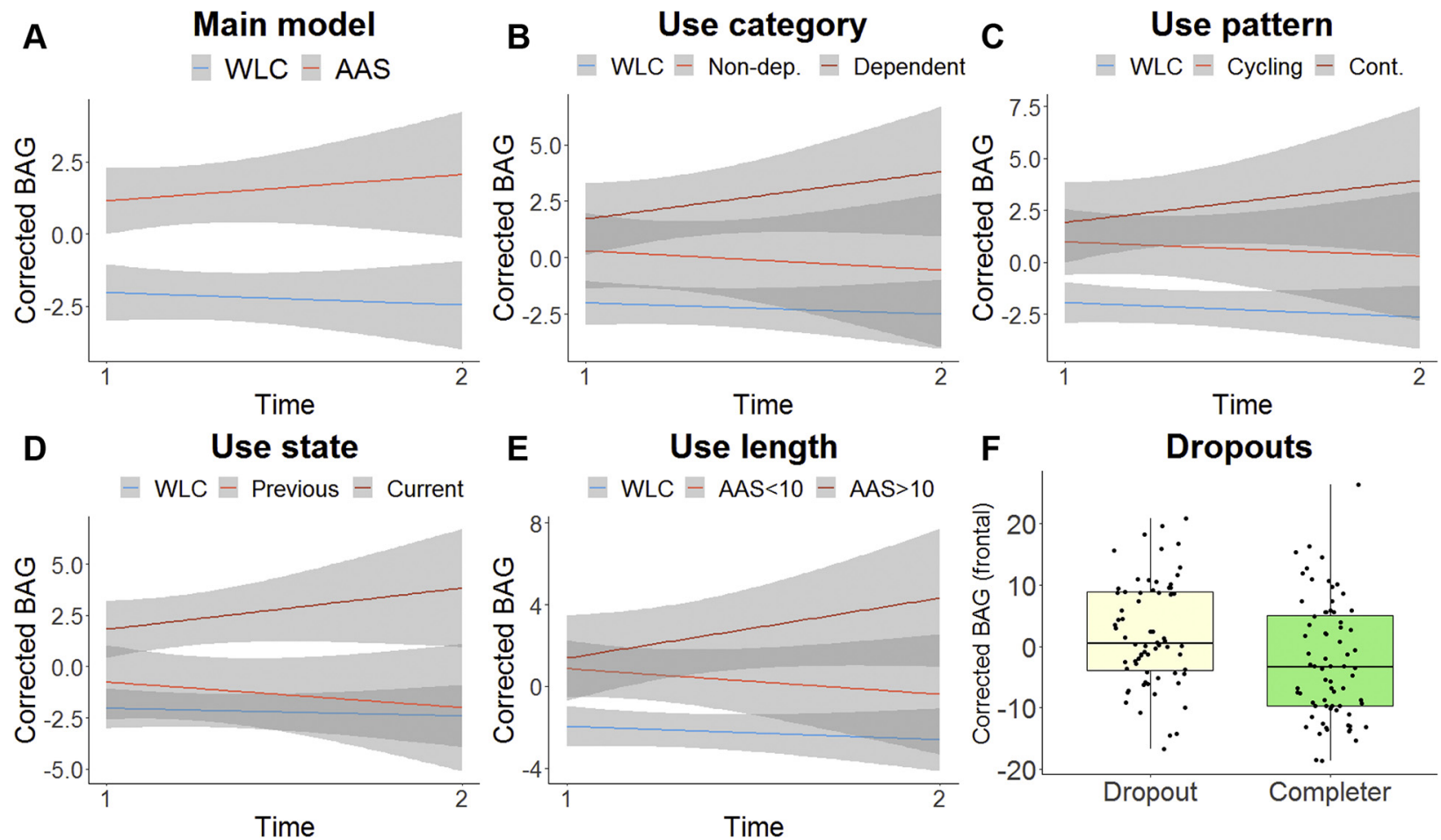

Figure 2. Brain age gap (BAG) in subgroups. Panels (A-E) show group $\times$ time (x-axis) interaction for corrected BAG (y-axis) of subgroups of participants with two scans approximately 3.5 years apart. Fitted lines were made with linear mixed effects-derived predicted values. Shaded gray areas represent $95 \%$ confidence intervals. Panel (F) shows a box plot of corrected BAG at baseline for participants who completed or dropped out of the study. Horizontal lines represent medians of samples. AAS, anabolic-androgenic steroid users; AAS $<10$, AAS users with $<10$ years of exposure; AAS $>10$, AAS users with $\geq 10$ years of exposure; Cont., continuous use; Non-dep., nondependent; WLC, weightlifting control subjects. 
Table 3. Main Model With Covariates

\begin{tabular}{|c|c|c|c|c|c|c|c|c|}
\hline & Full Brain & Frontal & Temporal & Insula & Cingulate & Parietal & Occipital & Subcortical \\
\hline \multicolumn{9}{|l|}{ Group AAS } \\
\hline Fixed-effects estimate $(\beta)$ & $3.631^{a}$ & $3.737^{b}$ & 2.458 & 1.997 & 2.568 & 1.836 & 1.571 & $3.516^{b}$ \\
\hline SE & 1.177 & 1.550 & 1.354 & 1.301 & 1.546 & 1.274 & 1.551 & 1.381 \\
\hline$t$ statistic & 3.085 & 2.411 & 1.815 & 1.535 & 1.662 & 1.441 & 1.013 & 2.547 \\
\hline FDR-corrected $p$ & .016 & .045 & .142 & .169 & .157 & .173 & .312 & .045 \\
\hline \multicolumn{9}{|l|}{ Time } \\
\hline Fixed-effects estimate $(\beta)$ & -0.194 & -0.751 & -0.727 & -1.046 & -1.291 & 0.510 & 1.302 & 0.536 \\
\hline SE & 0.627 & 0.916 & 0.693 & 0.672 & 0.846 & 0.698 & 0.891 & 0.673 \\
\hline$t$ statistic & -0.309 & -0.820 & -1.049 & -1.556 & -1.526 & 0.731 & 1.461 & 0.796 \\
\hline FDR-corrected $p$ & .758 & .534 & .534 & .392 & .392 & .534 & .392 & .534 \\
\hline \multicolumn{9}{|l|}{ Age } \\
\hline Fixed-effects estimate $(\beta)$ & -0.011 & -0.006 & -0.016 & 0.022 & 0.060 & -0.041 & -0.012 & -0.025 \\
\hline SE & 0.056 & 0.073 & 0.065 & 0.062 & 0.074 & 0.061 & 0.074 & 0.066 \\
\hline$t$ statistic & -0.198 & -0.076 & -0.253 & 0.353 & 0.820 & -0.669 & -0.168 & -0.374 \\
\hline FDR-corrected $p$ & .939 & .939 & .939 & .939 & .939 & .939 & .939 & .939 \\
\hline \multicolumn{9}{|l|}{$\mathrm{IQ}$} \\
\hline Fixed-effects estimate $(\beta)$ & 0.009 & -0.012 & -0.074 & 0.015 & -0.009 & $-0.101^{b}$ & $-0.152^{b}$ & 0.050 \\
\hline SE & 0.047 & 0.063 & 0.053 & 0.051 & 0.062 & 0.051 & 0.063 & 0.054 \\
\hline$t$ statistic & 0.194 & -0.187 & -1.389 & 0.300 & -0.140 & -1.986 & -2.434 & 0.935 \\
\hline FDR-corrected $p$ & .889 & .889 & .443 & .889 & .889 & .192 & .128 & .702 \\
\hline \multicolumn{9}{|l|}{ Substance Use } \\
\hline Fixed-effects estimate $(\beta)$ & -0.479 & -0.277 & -0.034 & 0.759 & -1.067 & -0.302 & 0.434 & $-1.476^{b}$ \\
\hline SE & 0.584 & 0.808 & 0.657 & 0.635 & 0.777 & 0.641 & 0.798 & 0.651 \\
\hline$t$ statistic & -0.821 & -0.342 & -0.052 & 1.195 & -1.373 & -0.472 & 0.544 & -2.266 \\
\hline FDR-corrected $p$ & .826 & .837 & .958 & .624 & .624 & .837 & .837 & .2 \\
\hline \multicolumn{9}{|l|}{ Depression } \\
\hline Fixed-effects estimate $(\beta)$ & -0.136 & -0.096 & -0.628 & -0.797 & -0.205 & -0.260 & -0.284 & -0.453 \\
\hline SE & 0.460 & 0.630 & 0.520 & 0.502 & 0.611 & 0.503 & 0.624 & 0.518 \\
\hline$t$ statistic & -0.296 & -0.152 & -1.209 & -1.589 & -0.335 & -0.516 & -0.455 & -0.874 \\
\hline FDR-corrected $p$ & .877 & .879 & .877 & .877 & .877 & .877 & .877 & .877 \\
\hline Observations & 217 & 217 & 217 & 217 & 217 & 217 & 217 & 217 \\
\hline Log Likelihood & -692.323 & -758.245 & -720.425 & -712.307 & -753.042 & -711.166 & -756.652 & -721.886 \\
\hline Akaike Information Criterion & $1,402.645$ & $1,534.491$ & $1,458.850$ & $1,442.614$ & $1,524.085$ & $1,440.331$ & $1,531.303$ & $1,461.772$ \\
\hline Bayesian Information Criterion & $1,433.065$ & $1,564.910$ & $1,489.270$ & $1,473.033$ & $1,554.504$ & $1,470.751$ & $1,561.722$ & $1,492.191$ \\
\hline
\end{tabular}

The upper section shows linear mixed-effects model results for estimates of brain age gaps for full brain and subregions. Group levels: weightlifting control subjects (reference) and AAS users.

AAS, anabolic-androgenic steroids; FDR, false discovery rate.

${ }^{a} p$ (uncorrected) $<.01$.

${ }^{b} p$ (uncorrected) $<.05$.

subregions, with most pronounced differences seen with longer exposure (Table S13).

\section{BAG Associated With Dropout}

About half $(56.7 \%)$ of the WLCs and $46.3 \%$ of the AAS users from TP1 participated at TP2. Frontal and cingulate BAG at baseline was significantly higher in participants who dropped out of the study compared with those with complete longitudinal data, whereas no significant differences were seen for other regions and the global BAG (Table 4).

\section{DISCUSSION}

Accumulating evidence suggests that prolonged AAS use harms the brain $(12,36,37,39,42,43)$. Using brain scans and brain age prediction based on an independent training set, we found evidence of higher relative global, frontal, temporal, occipital, and insular brain age in 130 male AAS users compared with 99 male WLCs. Furthermore, among AAS users, we found that long-term use and dependence were associated with higher relative brain age. Longitudinal analysis revealed no evidence of accelerated BAG over time in the overall AAS group; however, AAS users with more than 10 years of AAS exposure showed accelerated aging compared with WLCs, with a significant increase in BAG between TP1 and TP2 in this subgroup. These findings suggest that longterm high-dose AAS use may have adverse effects on brain aging, potentially linked to dependency and exaggerated use of AASs. 
Table 4. Baseline Brain Age Gap for Dropouts (After Time Point 1) and Completers Across Groups

\begin{tabular}{|c|c|c|c|c|c|c|c|c|}
\hline & Full Brain & Frontal & Temporal & Insula & Cingulate & Parietal & Occipital & Subcortical \\
\hline \multicolumn{9}{|c|}{ Dropouts and Completers } \\
\hline Estimate $(\beta)$ & -2.145 & $-4.321^{a}$ & 1.040 & 0.238 & $-3.799^{a}$ & -2.015 & -0.851 & 0.792 \\
\hline SE & 1.165 & 1.538 & 1.332 & 1.265 & 1.431 & 1.297 & 1.413 & 1.360 \\
\hline FDR-corrected $p$ & .181 & .036 & .642 & .852 & .036 & .246 & .642 & .642 \\
\hline \multicolumn{9}{|l|}{ Age } \\
\hline Estimate $(\beta)$ & 0.024 & 0.043 & 0.052 & 0.047 & 0.171 & 0.017 & -0.037 & 0.032 \\
\hline SE & 0.073 & 0.097 & 0.084 & 0.080 & 0.090 & 0.082 & 0.089 & 0.086 \\
\hline FDR-corrected $p$ & .839 & .839 & .839 & .839 & .839 & .839 & .839 & .839 \\
\hline Observations & 139 & 139 & 139 & 139 & 139 & 139 & 139 & 139 \\
\hline$R^{2}$ & .025 & .056 & .007 & .003 & .073 & .018 & .004 & .004 \\
\hline Adjusted $R^{2}$ & .011 & .042 & -.007 & -.012 & .059 & .003 & -.011 & -.011 \\
\hline Residual SE $(d f=136)$ & 6.867 & 9.065 & 7.852 & 7.457 & 8.431 & 7.642 & 8.327 & 8.015 \\
\hline$F_{2,136}$ & 1.749 & $4.046^{b}$ & 0.493 & 0.194 & $5.336^{a}$ & 1.229 & 0.268 & 0.241 \\
\hline
\end{tabular}

The upper section shows linear model results for brain age gap estimates for full brain and subregions. Dropout levels: dropouts (reference) and completers.

FDR, false discovery rate.

${ }^{a} p$ (uncorrected) $<.01$.

${ }^{b} p$ (uncorrected) $<.05$.

\section{AAS Use Associated With Apparent Brain Aging}

More evident brain aging in long-term AAS users is consistent with in vitro studies suggesting that various sorts of AASs might have neurotoxic effects (19-24) and recent findings of impaired cognitive performance $(12,39,40)$, smaller brain volumes (37), and metabolite abnormalities (36) in long-term AAS users. Older-appearing brains in AAS-dependent users compared with nondependent users is consistent with a megaanalysis pooling data from 23 cohorts, suggesting that dependence shares a common neural substrate across a range of substances, indicating smaller brain volumes and thinner cortex in dependent individuals relative to nondependent individuals (75). The group difference in global BAG suggests widespread effects, although regional models revealed significant differences in several regions, most pronounced frontally. Interestingly, the insula and part of the frontal cortex have been implicated in substance dependence (76-79), and our findings align with structural MRI studies showing reduced insular and frontal gray matter volume in drug users $(75,80)$.

AAS dependence, current use, and longer history of AASs of use were associated with higher BAG. The apparent difference in BAG between past and current AAS users should be regarded with caution and could be confounded by the considerable shorter duration of use among the past users. The links between AAS use and brain aging are likely complex and reflecting individual vulnerability, properties with the compounds being administered, and potential links to medically induced side effects. In line with this, users with $\geq 10$ years of AAS exposure or AAS dependence, which is characterized by more exaggerated use, the presence of psychological and/or medical side effects, and continued use despite negative impact on life $(1,15,40)$, showed the most prominent accelerated aging over time compared with WLCs.

Moreover, we found that AAS users who had dropped out of the study after TP1 had older-appearing brains in frontal and cingulate regions compared with those who completed the study. Hence, with a dropout rate of $49 \%$ in the total sample and $54 \%$ in the AAS user group, it is likely that our longitudinal findings are biased.

Some limitations should be noted. Whereas we included both cross-sectional and longitudinal data, the high dropout rate and nonrandom attrition might have limited the generalizability of the longitudinal models. This is in line with previous longitudinal studies of brain aging and dementia, showing that study dropout is associated with past worse executive and memory functioning (81) and MRI findings suggestive of higher future dementia risk (82). Furthermore, owing to the age distribution of the sample, the generalizability to the older AAS population is unclear. Moreover, while the total sample size is relatively large considering barriers of recruiting participants when studying clandestine and illegal behaviors, our sensitivity analyses resulted in small subsamples and estimates with high uncertainty. For instance, while previous users did not differ from WLCs, which could suggest part or full recovery after ceasing AAS use, larger follow-up studies of past users covering a wide age range are warranted to make plausible conclusions about recovery. It will also be important to study a potential link between long-term AAS use and white matter measures (e.g., measured using diffusion MRI) and, given the strong vascular effects of AAS $(27,83,84)$, measures of cerebral blood flow, and slowly progressive vascular pathology such as small-vessel disease.

The group differences could not be explained by general cognitive abilities, depression, or non-AAS substance use. Still, AAS use is commonly combined with a variety of drugs, such as aromatase inhibitors, human chorionic gonadotropin, tamoxifen, $5 \alpha$-reductase inhibitors, growth hormone, insulinlike growth factor, and dietary supplements, as well as narcotics and stimulants (85). In addition, the intricate administration pattern of AASs typically includes different doses and stacking of multiple classes of AASs with different molecular and cellular effects (86). Such complexity makes it extremely difficult to distinguish the unique contributions of single factors 
on measures of brain health and behavior. Moreover, a range of psychological and medical effects linked to AAS use might influence brain health (15). Hence, future interdisciplinary studies are needed to better understand mechanisms linking AAS use and brain aging.

In conclusion, in line with mounting evidence of adverse health effects of AAS use, using brain age prediction, we found evidence of increased apparent brain aging in long-term highdose AAS users, seemingly linked to dependency and exaggerated use of AASs.

\section{ACKNOWLEDGMENTS AND DISCLOSURES}

This research was funded by the South-Eastern Norway Regional Health Authority (Grant Nos. 2013087, 2016049, 2017025, and 2018075 [to AB]) and by internal research grants from the Division on Mental Health and Addiction (to AB). LTW is funded by the European Research Council under the European Union's Horizon 2020 research and innovation program (ERC Starting Grant No. 802998), the Research Council of Norway (Grant Nos. 249795, 273345, and 298646), the South-Eastern Norway Regional Health Authority (Grant No. 2019101), and the Department of Psychology, University of Oslo, Norway. TK is funded by the Research Council of Norway (Grant No. 276082).

Datasets for model training were partly gathered from public resources. For CamCAN, data collection and sharing for this project were provided by the Cambridge Centre for Ageing and Neuroscience (CamCAN) (https://camcanarchive.mrc-cbu.cam.ac.uk/dataaccess). CamCAN funding was provided by the UK Biotechnology and Biological Sciences Research Council (Grant No. BB/H008217/1) together with support from the UK Medical Research Council and University of Cambridge. For DLBC, datasets were obtained from http:// fcon_1000.projects.nitrc.org. For HCP, data were provided in part by the Human Connectome Project (HCP), WU-Minn Consortium (principal investigators David Van Essen and Kamil Ugurbil; Grant No. 1U54MH091657) funded by the 16 National Institutes of Health $(\mathrm{NIH})$ institutes and centers that support the NIH Blueprint for Neuroscience Research and by the McDonnell Center for Systems Neuroscience at Washington University. For details, see https://www.humanconnectome.org/study/hcp-young-adult/document/hcpcitations. For ds000222, datasets were obtained from the OpenfMRI database (https://openfmri.org). For IXI, datasets were obtained from http://braindevelopment.org/ixi-dataset. For OASIS, datasets were obtained from http://www.oasis-brains.org. The study was supported by National Institutes of Health Grant Nos. P50 AG05681, P01 AG03991, R01 AG021910, P50 MH071616, U24 RR021382, and R01 MH56584.40. For SALD, datasets were obtained from http://fcon_1000.projects.nitrc.org. For STROKEMRI, data collection was supported by the Research Council of Norway (Grant No. 249795 and 248238), the South-Eastern Norway Regional Health Authority (Grant Nos. 2014097, 2015044, 2015073, and 2016083), and the Norwegian Extra Foundation for Health and Rehabilitation (Grant No. 2015/FO5146). The funding organizations had no role in the design or conduct of the study; in the collection, analysis, or interpretation of data; or in the preparation, review, or approval of the manuscript.

A previous version of this article was published as a preprint on medRxiv: https://www.medrxiv.org/content/10.1101/2020.08.19.20177832v1.article-info.

The authors report no biomedical financial interests or potential conflicts of interest.

\section{ARTICLE INFORMATION}

From the Anabolic Androgenic Steroid Research Group (AB, LEH, SK), Section for Clinical Addiction Research, Division of Mental Health and Addiction, Oslo University Hospital; Norwegian Centre for Mental Disorders Research (NORMENT) (TK, LTW), Institute of Clinical Medicine, University of Oslo, and Division of Mental Health and Addiction, Oslo University Hospital; Norwegian Doping Control Laboratory (IRH), Oslo University Hospital; Department of Psychology (LTW) and K.G. Jebsen Center for Neurodevelopmental Disorders (LTW), University of Oslo, Oslo, Norway; and Department of Psychiatry and Psychotherapy (TK), University of Tübingen, Tübingen, Germany.
Address correspondence to Astrid Bjørnebekk, Ph.D., at askrbj@ous-hf. no.

Received Sep 3, 2020; revised Dec 2, 2020; accepted Jan 4, 2021.

Supplementary material cited in this article is available online at https:// doi.org/10.1016/j.bpsc.2021.01.001.

\section{REFERENCES}

1. Kanayama G, Hudson JI, Pope HG Jr (2009): Features of men with anabolic-androgenic steroid dependence: A comparison with nondependent AAS users and with AAS nonusers. Drug Alcohol Depend 102:130-137.

2. Bhasin S, Storer TW, Berman N, Callegari C, Clevenger B, Phillips J, et al. (1996): The effects of supraphysiologic doses of testosterone on muscle size and strength in normal men. N Engl J Med 335:1-7.

3. Pope HG Jr, Kouri EM, Hudson JI (2000): Effects of supraphysiologic doses of testosterone on mood and aggression in normal men: A randomized controlled trial. Arch Gen Psychiatry 57:133-140.. discussion 155-156.

4. Thiblin I, Runeson B, Rajs J (1999): Anabolic androgenic steroids and suicide. Ann Clin Psychiatry 11:223-231.

5. Basaria S, Wahlstrom JT, Dobs AS (2001): Anabolic-androgenic steroid therapy in the treatment of chronic diseases. J Clinical Endocrinol Metab 86:5108-5117.

6. Shahidi NT (2001): A review of the chemistry, biological action, and clinical applications of anabolic-androgenic steroids. Clin Ther 23:1355-1390.

7. Pomerantz SM, Fox TO, Sholl SA, Vito CC, Goy RW (1985): Androgen and estrogen receptors in fetal rhesus monkey brain and anterior pituitary. Endocrinology 116:83-89.

8. Simerly RB, Chang C, Muramatsu M, Swanson LW (1990): Distribution of androgen and estrogen receptor mRNA-containing cells in the rat brain: An in situ hybridization study. J Comp Neurol 294:76-95.

9. Mitra SW, Hoskin E, Yudkovitz J, Pear L, Wilkinson HA, Hayashi S, et al. (2003): Immunolocalization of estrogen receptor beta in the mouse brain: Comparison with estrogen receptor alpha. Endocrinology 144:2055-2067.

10. Reyes-Fuentes A, Veldhuis JD (1993): Neuroendocrine physiology of the normal male gonadal axis. Endocrinol Metab Clin North Am 22:93124.

11. Pope HG Jr, Wood RI, Rogol A, Nyberg F, Bowers L, Bhasin S (2014): Adverse health consequences of performance-enhancing drugs: An Endocrine Society scientific statement. Endocr Rev 35:341-375.

12. Bjørnebekk A, Westlye LT, Walhovd KB, Jørstad ML, Sundseth $\varnothing \varnothing$, Fjell AM (2019): Cognitive performance and structural brain correlates in long-term anabolic-androgenic steroid exposed and nonexposed weightlifters. Neuropsychology 33:547-559.

13. Brower KJ (2002): Anabolic steroid abuse and dependence. Curr Psychiatry Rep 4:377-387.

14. Ip EJ, Barnett MJ, Tenerowicz MJ, Perry PJ (2011): The Anabolic 500 survey: Characteristics of male users versus nonusers of anabolicandrogenic steroids for strength training. Pharmacotherapy 31:757766.

15. Kanayama G, Brower KJ, Wood RI, Hudson JI, Pope HG Jr (2009): Anabolic-androgenic steroid dependence: An emerging disorder. Addiction 104:1966-1978.

16. Chandler M, McVeigh J (2013): Steroids and Image Enhancing Drugs 2013 Survey Results. Liverpool, UK: Centre for Public Health, Liverpool John Moores University.

17. Brower KJ (1997): Withdrawal from anabolic steroids. Curr Ther Endocrinol Metab 6:338-343.

18. Kashkin KB, Kleber HD (1989): Hooked on hormones? An anabolic steroid addiction hypothesis. JAMA 262:3166-3170.

19. Hammond J, Le Q, Goodyer C, Gelfand M, Trifiro M, LeBlanc A (2001): Testosterone-mediated neuroprotection through the androgen receptor in human primary neurons. J Neurochem 77:1319-1326.

20. Nguyen TV, Yao M, Pike CJ (2005): Androgens activate mitogenactivated protein kinase signaling: Role in neuroprotection. J Neurochem 94:1639-1651. 
21. Basile JR, Binmadi NO, Zhou H, Yang YH, Paoli A, Proia P (2013): Supraphysiological doses of performance enhancing anabolicandrogenic steroids exert direct toxic effects on neuron-like cells. Front Cell Neurosci 7:69.

22. Caraci F, Pistara V, Corsaro A, Tomasello F, Giuffrida ML, Sortino MA et al. (2011): Neurotoxic properties of the anabolic androgenic steroids nandrolone and methandrostenolone in primary neuronal cultures. J Neurosci Res 89:592-600.

23. Estrada M, Varshney A, Ehrlich BE (2006): Elevated testosterone induces apoptosis in neuronal cells. J Biol Chem 281:25492-25501.

24. Westlye ET, Lundervold A, Rootwelt H, Lundervold AJ, Westlye LT (2011): Increased hippocampal default mode synchronization during rest in middle-aged and elderly APOE $\varepsilon 4$ carriers: Relationships with memory performance. J Neurosci 31:7775-7783.

25. Zelleroth S, Nylander E, Nyberg F, Gronbladh A, Hallberg M (2019): Toxic impact of anabolic androgenic steroids in primary rat cortical cell cultures. Neuroscience 397:172-183.

26. Orlando R, Caruso A, Molinaro G, Motolese M, Matrisciano F, Togna G, et al. (2007): Nanomolar concentrations of anabolicandrogenic steroids amplify excitotoxic neuronal death in mixed mouse cortical cultures. Brain Res 1165:21-29.

27. Baggish AL, Weiner RB, Kanayama G, Hudson JI, Lu MT, Hoffmann U, et al. (2017): Cardiovascular toxicity of illicit anabolic-androgenic steroid use. Circulation 135:1991-2002.

28. Nottin S, Nguyen LD, Terbah M, Obert P (2006): Cardiovascular effects of androgenic anabolic steroids in male bodybuilders determined by tissue Doppler imaging. Am J Cardiol 97:912-915.

29. Kanayama G, Hudson JI, DeLuca J, Isaacs S, Baggish A, Weiner R, et al. (2015): Prolonged hypogonadism in males following withdrawal from anabolic-androgenic steroids: An under-recognized problem. Addiction 110:823-831.

30. Rasmussen JJ, Selmer C, Ostergren PB, Pedersen KB, Schou M, Gustafsson F, et al. (2016): Former abusers of anabolic androgenic steroids exhibit decreased testosterone levels and hypogonadal symptoms years after cessation: A case-control study. PLoS One 11: e161208.

31. Gheshlaghi F, Piri-Ardakani MR, Masoumi GR, Behjati M, Paydar P (2015): Cardiovascular manifestations of anabolic steroids in association with demographic variables in body building athletes. J Res Med Sci 20:165-168.

32. Rasmussen JJ, Schou M, Selmer C, Johansen ML, Gustafsson F, Frystyk J, et al. (2017): Insulin sensitivity in relation to fat distribution and plasma adipocytokines among abusers of anabolic androgenic steroids. Clin Endocrinol (Oxf) 87:249-256.

33. Modlinski R, Fields KB (2006): The effect of anabolic steroids on the gastrointestinal system, kidneys, and adrenal glands. Curr Sports Med Rep 5:104-109.

34. de Lange AG, Anaturk M, Suri S, Kaufmann T, Cole JH, Griffanti L, et al. (2020): Multimodal brain-age prediction and cardiovascular risk: The Whitehall II MRI sub-study. Neurolmage 222:117292.

35. Gorelick PB, Scuteri A, Black SE, Decarli C, Greenberg SM ladecola C, et al. (2011): Vascular contributions to cognitive impairment and dementia: A statement for healthcare professionals from the American Heart Association/American Stroke Association. Stroke 42:2672-2713.

36. Kaufman MJ, Janes AC, Hudson JI, Brennan BP, Kanayama G, Kerrigan AR, et al. (2015): Brain and cognition abnormalities in longterm anabolic-androgenic steroid users. Drug Alcohol Depend $1: 47-56$.

37. Bjørnebekk A, Walhovd KB, Jørstad ML, Due-Tønnessen P, Hullstein IR, Fjell AM (2017): Structural brain imaging of long-term anabolic-androgenic steroid users and nonusing weightlifters. Biol Psychiatry 82:294-302.

38. Westlye LT, Kaufmann T, Alnaes D, Hullstein IR, Bjørnebekk A (2017): Brain connectivity aberrations in anabolic-androgenic steroid users. Neurolmage Clin 13:62-69.

39. Kanayama G, Kean J, Hudson JI, Pope HG Jr (2013): Cognitive deficits in long-term anabolic-androgenic steroid users. Drug Alcohol Depend 130:208-214.
40. Hauger LE, Westlye LT, Bjornebekk A (2020): Anabolic androgenic steroid dependence is associated with executive dysfunction. Drug Alcohol Depend 208:107874.

41. Heffernan TM, Battersby L, Bishop P, O’Neill TS (2015): Everyday Memory Deficits Associated with Anabolic-Androgenic Steroid Use in Regular Gymnasium Users. Open Pychiatry J 9:1-6.

42. Kaufman MJ, Kanayama G, Hudson JI, Pope HG Jr (2019): Supraphysiologic-dose anabolic-androgenic steroid use: A risk factor for dementia? Neurosci Biobehav Rev 100:180-207.

43. Pomara C, Neri M, Bello S, Fiore C, Riezzo I, Turillazzi E (2015): Neurotoxicity by synthetic androgen steroids: Oxidative stress, apoptosis, and neuropathology: A review. Curr Neuropharmacol 13:132-145.

44. Hall RC, Chapman MJ (2005): Psychiatric complications of anabolic steroid abuse. Psychosomatics 46:285-290.

45. Hauger LE, Sagoe D, Vaskinn A, Arnevik EA, Leknes S, Jørstad ML, et al. (2019): Anabolic androgenic steroid dependence is associated with impaired emotion recognition. Psychopharmacology (Berl) 236:2667-2676.

46. Etkin A (2019): A reckoning and research agenda for neuroimaging in psychiatry. Am J Psychiatry 176:507-511.

47. Dosenbach NU, Nardos B, Cohen AL, Fair DA, Power JD, Church JA, et al. (2010): Prediction of individual brain maturity using fMRI. Science 329:1358-1361.

48. Franke K, Ziegler G, Kloppel S, Gaser C, Alzheimer's Disease Neuroimaging I (2010): Estimating the age of healthy subjects from T1weighted MRI scans using kernel methods: Exploring the influence of various parameters. Neurolmage 50:883-892.

49. Cole JH, Franke K (2017): Predicting age using neuroimaging: innovative brain ageing biomarkers. Trends Neurosci 40:681-690.

50. Cole JH, Poudel RPK, Tsagkrasoulis D, Caan MWA, Steves C, Spector TD, et al. (2017): Predicting brain age with deep learning from raw imaging data results in a reliable and heritable biomarker. Neurolmage 163:115-124.

51. Liem F, Varoquaux G, Kynast J, Beyer F, Kharabian Masouleh S, Huntenburg JM, et al. (2017): Predicting brain-age from multimodal imaging data captures cognitive impairment. Neurolmage 148:179188.

52. Cole JH, Ritchie SJ, Bastin ME, Valdes Hernandez MC, Munoz Maniega S, Royle N, et al. (2018): Brain age predicts mortality. Mo Psychiatry 23:1385-1392.

53. Lebedeva AK, Westman E, Borza T, Beyer MK, Engedal K, Aarsland D, et al. (2017): MRI-based classification models in prediction of mild cognitive impairment and dementia in late-life depression. Front Aging Neurosci 9:13.

54. Kaufmann T, van der Meer D, Doan NT, Schwarz E, Lund MJ, Agartz I, et al. (2019): Common brain disorders are associated with heritable patterns of apparent aging of the brain. Nat Neurosci 22:1617-1623.

55. Steffener J, Habeck C, O'Shea D, Razlighi Q, Bherer L, Stern Y (2016): Differences between chronological and brain age are related to education and self-reported physical activity. Neurobiol Aging 40:138-144.

56. Ersche KD, Jones PS, Williams GB, Robbins TW, Bullmore ET (2013): Cocaine dependence: A fast-track for brain ageing? Mol Psychiatry 18:134-135.

57. Nakama H, Chang L, Fein G, Shimotsu R, Jiang CS, Ernst T (2011): Methamphetamine users show greater than normal age-related cortical gray matter loss. Addiction 106:1474-1483.

58. Guggenmos M, Schmack K, Sekutowicz M, Garbusow M, Sebold M, Sommer C, et al. (2017): Quantitative neurobiological evidence for accelerated brain aging in alcohol dependence. Transl Psychiatry 7:1279.

59. Bachi K, Sierra S, Volkow ND, Goldstein RZ, Alia-Klein N (2017): Is biological aging accelerated in drug addiction? Curr Opin Behav Sci 13:34-39.

60. de Lange AG, Barth C, Kaufmann T, Maximov II, van der Meer D, Agartz I, et al. (2020): Women's brain aging: Effects of sex-hormone exposure, pregnancies, and genetic risk for Alzheimer's disease. Hum Brain Mapp 41:5141-5150. 
61. Dale AM, Fischl B, Sereno MI (1999): Cortical surface-based analysis: I. Segmentation and surface reconstruction. Neurolmage 9:179-194.

62. Glasser MF, Coalson TS, Robinson EC, Hacker CD, Harwell J, Yacoub E, et al. (2016): A multi-modal parcellation of human cerebral cortex. Nature 536:171-178.

63. Richard G, Kolskar K, Sanders AM, Kaufmann T, Petersen A, Doan NT, et al. (2018): Assessing distinct patterns of cognitive aging using tissue-specific brain age prediction based on diffusion tensor imaging and brain morphometry. PeerJ 6:e5908.

64. Le TT, Kuplicki RT, McKinney BA, Yeh HW, Thompson WK, Paulus MP, et al. (2018): A nonlinear simulation framework supports adjusting for age when analyzing BrainAGE. Front Aging Neurosci 10:317.

65. de Lange AG, Cole JH (2020): Commentary: Correction procedures in brain-age prediction. Neurolmage Clin 26:102229.

66. Saunders JB, Aasland OG, Babor TF, de la Fuente JR, Grant M (1993): Development of the Alcohol Use Disorders Identification Test (AUDIT): WHO collaborative project on early detection of persons with harmful alcohol consumption-II. Addiction 88:791-804.

67. Berman AH, Bergman H, Palmstierna T, Schlyter F (2005): Evaluation of the Drug Use Disorders Identification Test (DUDIT) in criminal justice and detoxification settings and in a Swedish population sample. Eur Addict Res 11:22-31.

68. Hauger LE, Westlye LT, Fjell AM, Walhovd KB, Bjornebekk A (2019): Structural brain characteristics of anabolic-androgenic steroid dependence in men. Addiction 114:1405-1415.

69. Pope HG Jr, Katz DL (1994): Psychiatric and medical effects of anabolic-androgenic steroid use: A controlled study of 160 athletes. Arch Gen Psychiatry 51:375-382.

70. Wechsler D (1999): Wechsler Abbreviated Scale of Intelligens. San Antonio, TX: Psychological Corporation.

71. Hullstein IR, Malerod-Fjeld H, Dehnes Y, Hemmersbach P (2015): Black market products confiscated in Norway 2011-2014 compared to analytical findings in urine samples. Drug Test Anal 7:1025-1029.

72. R Core Team (2014): R: A Language and Environment for Statistical Computing. Vienna, Austria: R Foundation for Statistical Computing.

73. Bates D, Mächler M, Bolker B, Walker S (2015): Fitting linear mixedeffects models using Ime4. J Stat Softw 67:1-48.

74. Benjamini $Y$, Hochberg $Y$ (1995): Controlling the false discovery rate: A practical and powerful approach to multiple testing. J R Stat Soc Ser B $57: 289-300$.
75. Mackey S, Allgaier N, Chaarani B, Spechler P, Orr C, Bunn J, et al. (2019): Mega-analysis of gray matter volume in substance dependence: General and substance-specific regional effects. Am J Psychiatry 176:119-128.

76. Everitt BJ, Robbins TW (2005): Neural systems of reinforcement for drug addiction: From actions to habits to compulsion. Nat Neurosci 8:1481-1489.

77. Goldstein RZ, Craig AD, Bechara A, Garavan H, Childress AR, Paulus MP, et al. (2009): The neurocircuitry of impaired insight in drug addiction. Trends Cogn Sci 13:372-380.

78. Koob GF, Volkow ND (2010): Neurocircuitry of addiction. Neuropsychopharmacology 35:217-238.

79. Droutman V, Read SJ, Bechara A (2015): Revisiting the role of the insula in addiction. Trends Cogn Sci 19:414-420.

80. Ersche KD, Williams GB, Robbins TW, Bullmore ET (2013): Metaanalysis of structural brain abnormalities associated with stimulant drug dependence and neuroimaging of addiction vulnerability and resilience. Curr Opin Neurobiol 23:615-624.

81. Sünkel U, Heinzel S, Thaler A-K, Metzger F, Eschweiler G, LiepeltScarfone I, et al. (2016): Characteristics of dropouts in a longitudinal study of individuals at risk for a neurodegenerative disorder. International Congress of Parkinson's Disease and Movement Disorders, June, Berlin, Germany.

82. Glymour MM, Chene G, Tzourio C, Dufouil C (2012): Brain MRI markers and dropout in a longitudinal study of cognitive aging: The Three-City Dijon Study. Neurology 79:1340-1348.

83. Rasmussen JJ, Schou M, Madsen PL, Selmer C, Johansen ML, Hovind P, et al. (2018): Increased blood pressure and aortic stiffness among abusers of anabolic androgenic steroids: Potential effect of suppressed natriuretic peptides in plasma? J Hypertens 36:277-285.

84. Vanberg P, Atar D (2010): Androgenic anabolic steroid abuse and the cardiovascular system. Handb Exp Pharmacol 2010:411-457.

85. Sagoe D, McVeigh J, Bjornebekk A, Essilfie MS, Andreassen CS, Pallesen S (2015): Polypharmacy among anabolic-androgenic steroid users: A descriptive metasynthesis. Subst Abuse Treat Prev Policy 10:12.

86. Penatti CA, Porter DM, Henderson LP (2009): Chronic exposure to anabolic androgenic steroids alters neuronal function in the mammalian forebrain via androgen receptor- and estrogen receptor-mediated mechanisms. J Neurosci 29:12484-12496. 